\title{
VsN, a Reliability-index of Shear-wave Measurement in Sonoelastography, Is Useful for the Diagnosis of Thyroid Tumor Malignancy
}

\author{
TOMOO JIKUZONO ${ }^{1,2}$, OSAMU ISHIBASHI $^{1,2}$, SHOKO KURE $^{3}$, \\ CHIAKI ITOH $^{4}$, TETSU YAMADA ${ }^{5}$ and IWAO SUGITANI ${ }^{1}$ \\ ${ }^{1}$ Department of Endocrine Surgery, Nippon Medical School, Tokyo, Japan; \\ ${ }^{2}$ Laboratory of Biological Macromolecules, Department of Applied Life Sciences, \\ Graduate School of Life \& Environmental Sciences, Osaka Prefecture University, Osaka, Japan; \\ ${ }^{3}$ Department of Integrated Diagnostic Pathology, Nippon Medical School, Tokyo, Japan; \\ ${ }^{4}$ Dynacom Company, Ltd, Chiba, Japan; \\ ${ }^{5}$ Department of Endocrine Surgery, Kanaji Thyroid Hospital, Tokyo, Japan
}

\begin{abstract}
Background/Aim: Shear wave measurement $(S W M)$ is a new elastography modality that quantifies the shear wave velocity (Vs) and the percentage of the net effective shear wave velocity $(V s N)$. This study examined whether these parameters could be used to differentiate between malignant and benign thyroid tumors. Patients and Methods: The study of SWM enrolled 111 patients (133 nodules) who underwent thyroid surgery. Overall, 61 nodules were diagnosed as benign and 72 as malignant, of which 68 nodules were diagnosed as papillary thyroid carcinoma (PTC) and 4 as follicular thyroid carcinoma (FTC). Results: A preoperative SWM revealed that Vs was significantly higher and VSN significantly lower in the PTC compared to the benign nodules. The VsN of FTC was significantly lower than that of follicular adenoma. Multivariate analysis revealed that VsN significantly correlated with nodule malignancy. Conclusion: SWM parameters, especially $V s N$, can potentially differentiate between benign and malignant thyroid nodules noninvasively.
\end{abstract}

This article is freely accessible online.

Correspondence to: Osamu Ishibashi, Laboratory of Biological Macromolecules, Department of Applied Life Sciences, Graduate School of Life and Environmental Sciences, Osaka Prefecture University, Sakai 599-8531, Japan. Tel: +81 722549474, e-mail: ishibashi@biochem.osakafu-u.ac.jp

Key Words: Shear wave measurement, the percentage of the net amount of effective shear wave velocity, thyroid nodules, papillary thyroid carcinoma, follicular thyroid carcinoma.
Shear wave imaging (SWI), a type of ultrasound elasticity imaging, is a method that can provide quantitative information about tissue stiffness (1). So far, studies have found that shear moduli are highly correlated with the pathological status of human tissue, such as liver fibrosis (2, 3 ). There are also reports that SWI can be used to diagnose abnormal structures in the thyroid, such as benign and malignant nodules (4-7), lymph node metastasis (8), and chronic thyroiditis (9-12). Shear moduli can be investigated by measuring the velocity of the shear wave propagation (Vs) in a tissue region. An improved SWI, named shear wave measurement (SWM), has recently been developed (13). SWM can be classified as a point shear wave speed measurement, according to the guidelines of the World Federation for Ultrasound in Medicine and Biology (1), providing a unique reliability indicator, which is the percentage of the net effective shear wave velocity (VsN). VsN indicates the percentage of correctly detected measurements, thus, it is often used to evaluate the reliability of the Vs values obtained (14). Vs values are excluded if they meet any of the following criteria: i) negative Vs, ii) Vs outside a defined range, and iii) phase fluctuation detected at a particular depth.

Papillary thyroid carcinoma (PTC) is the most common thyroid carcinoma and is defined by distinct nuclear features, including alterations of nuclear size and shape, chromatin pattern and nuclear membrane irregularities $(15,16)$. It is easy to diagnose PTC using fine needle aspiration cytology preoperatively, although there are exceptions. Follicular thyroid carcinoma (FTC) is a thyroid carcinoma with follicular differentiation. Unlike PTC, FTC has no cytological features of malignancy $(15,16)$. Therefore, it is difficult or impossible to differentiate between FTC and 
Table I. Baseline characteristics of the 133 nodules studied.

\begin{tabular}{lcccc}
\hline Variables & $\begin{array}{c}\text { Benign nodules }(\mathrm{n}=61) \\
\text { (Numbers or mean } \pm \text { SD) }\end{array}$ & $\begin{array}{c}\text { Malignant nodules }(\mathrm{n}=72) \\
\text { (Numbers or mean } \pm \text { SD) }\end{array}$ & $\begin{array}{c}p \text {-Value } \\
\text { (Numbers or mean } \pm \text { SD) }\end{array}$ & $\begin{array}{r}\text { Total nodules }(\mathrm{n}=133) \\
\text { Age (years, range) }\end{array}$ \\
Gender (Female/Male) & $47.6 \pm 15.9(17-81)$ & $49.1 \pm 14.2(16-83)$ & 0.53 & $48.4 \pm 15.0(16-83)$ \\
Nodule size (mm, range) & $51 / 10$ & $55 / 17$ & 0.31 & $106 / 27$ \\
Chronic thyroiditis (Presence/Absence) & $81.7 \pm 13.2(7-64)$ & $13.4 \pm 9.0(2-62)$ & $<0.0001$ & $21.8 \pm 14.4(2-64)$ \\
\hline
\end{tabular}

follicular adenoma (FA), a benign follicular tumor, using fine needle aspiration cytology.

This study investigated whether $\mathrm{Vs}$ and $\mathrm{VsN}$ can differentially detect malignant thyroid nodules, including FTC.

\section{Patients and Methods}

Patients. In this study, we analyzed 111 patients who underwent SWM before surgery at Kanaji Thyroid Hospital between April 2019 and March 2020. We obtained informed consent from all patients and approval for this study from the Ethics Committee of Kanaji Thyroid Hospital (approval no. 15). The study was performed in accordance with the ethical guidelines of the Helsinki Declaration. The study enrolled 111 patients ( 88 females, 23 males) with 133 thyroid nodules, which included 61 benign and 72 malignant nodules (Table I and Figure 1). All of the histopathological diagnoses were determined independently of the ultrasound diagnosis. All nodules were also diagnosed preoperatively by cytology, based on the reporting format of the 7th edition of the Thyroid Cancer Treatment Protocol. The latter follows the Bethesda system for reporting thyroid cytopathology (17-19), which is an international standard format for cytology reporting, with slight modifications to suit the situation in Japan. The pathological classification of thyroid nodules was conducted according to the World Health Organization classification $(15,16)$. The proportions of the individual thyroid tumor types classified here are consistent with the reported thyroid tumor statistics (20). None of the nodules revealed an obvious distant metastasis in the preoperative examination. SWM was performed after hospitalization for surgical purposes as part of the preoperative screening test. Therefore, patients who underwent SWM were chosen at random.

Ultrasound examination. To evaluate the thyroid nodules preoperatively, ultrasound examination was performed using a FUJIFILM Healthcare ARIETTA 850 (FUJIFILM Healthcare, Tokyo, Japan) with an L64 linear ultrasound probe (5-18 MHz), and SWM data were obtained. For SWM on B-mode ultrasound images, a $10 \times 5 \mathrm{~mm}$ thyroid nodule region was set as the region of interest. Multiple shear waves were generated in the rectangular area at random and detected by sonographic detection pulses, and the numerical values of the SWM were displayed. Unless otherwise specified, five separate measurements were made at the same point to calculate average Vs and $\mathrm{VsN}$ values. In SWM, Vs and VsN are expressed in $\mathrm{m} / \mathrm{s}$ and $\%$, respectively.

Statistical analysis. The following parameters were obtained from the patients' medical records: i) age, ii) sex, iii) nodule size, iv) chronic thyroiditis (presence or absence of anti-thyroglobulin

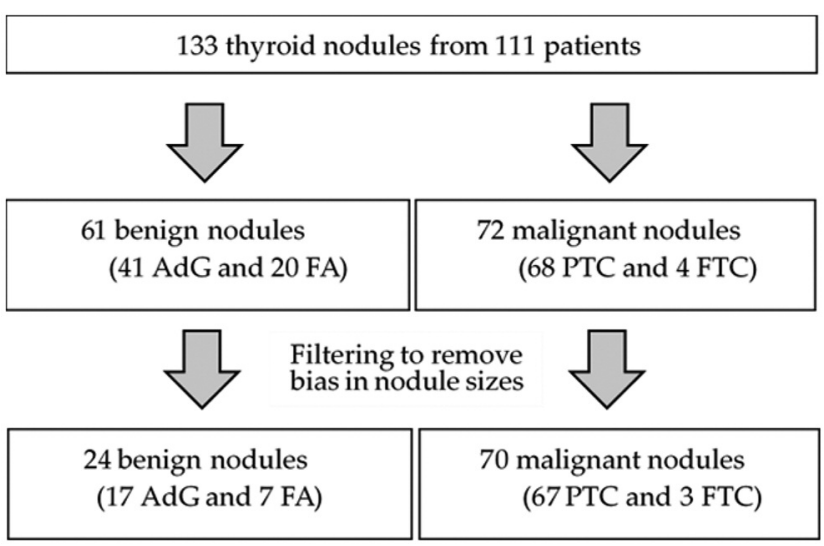

Figure 1. Flowchart of patient selection. PTC: Papillary thyroid carcinoma; FTC: follicular thyroid carcinoma; AdG: adenomatous goitre; FA: follicular adenoma.

antibody), v) lymph node metastasis (presence or absence) and vi) state of malignancy or not. The background characteristics of the benign and malignant groups were compared using the MannWhitney $U$-test. The Vs and VsN of the four groups [PTC, FTC, adenomatous goitre (AdG), and FA] were compared using the Kruskal-Wallis test, followed by a post-hoc Steel-Dwass test. The cut-off values of $\mathrm{Vs}$ and $\mathrm{VsN}$ were determined from Receiver Operating Characteristic (ROC) curve analysis and the area under the curve (AUC) was calculated. Correlation analyses were conducted between either Vs or VsN and the continuous parameters using Spearman's rank correlation coefficient, whereas the MannWhitney $U$-test was applied for categorical parameters. Multivariate linear regression analysis of VsN was performed to identify the clinical factors associated with this index. We checked the variance inflation factor (VIF), a measure of the amount of multicollinearity in a set of multiple regression variables, to examine multicollinearity among variables. The upper limit of the VIF indicating harmful collinearity was set at 10 . All statistical analyses to this point were performed using EZR (http://www.jichi.ac.jp/ saitama-sct/SaitamaHP.files/statmed.html), a modified version of $R$ commander (ver. 1.6-3) (R Foundation for Statistical Computing, Vienna, Austria), designed for statistical functions frequently used in biostatistics (21).

For subsequent statistical analysis, R, ver. 3, was used (22). We used psych, ver. 1.9.12.31, for the correlation matrix (23) and plsdepot, ver. 0.1.17, for the partial least squares (PLS) regression analysis (24). For the least absolute shrinkage and selection operator 

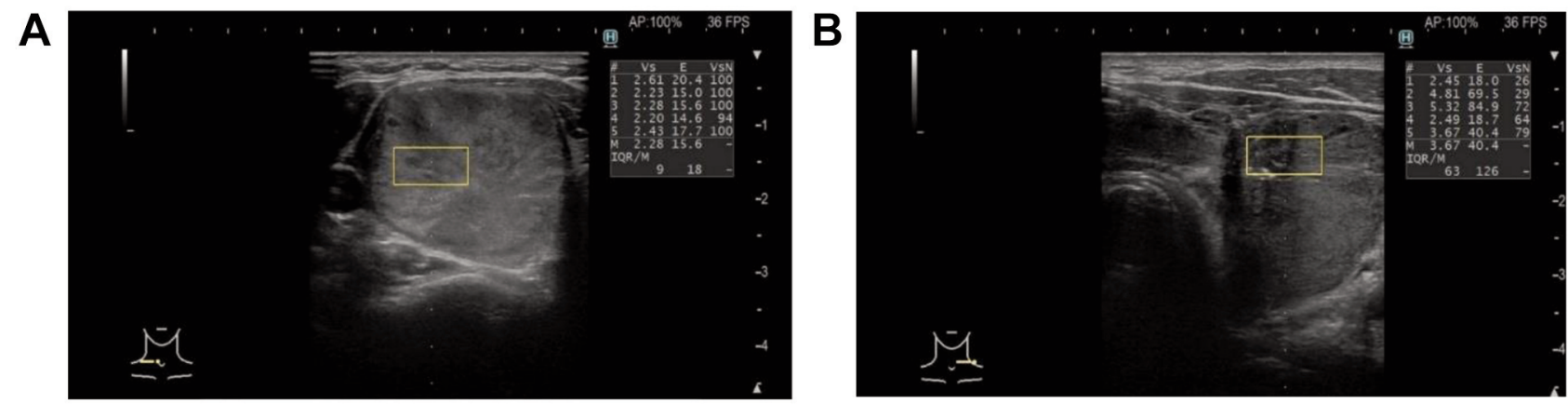

Figure 2. Representative SWM images of high-elasticity and low-elasticity nodules. (A) A 39-year-old woman had a 34.3-mm-diameter nodule in the right thyroid lobe. This nodule was pathologically diagnosed as follicular adenoma (FA). Shear wave velocity (Vs) and the net amount of effective shear wave velocity $(V s N)$ are $2.35 \mathrm{~m} / \mathrm{s}$ and $98.8 \%$, respectively. (B) An 80-year-old male had a 20.0-mm-diameter nodule in the left thyroid lobe. This nodule was pathologically diagnosed as papillary thyroid carcinoma (PTC). Vs and VsN are $3.75 \mathrm{~m} / \mathrm{s}$ and $54.0 \%$, respectively.

(LASSO) regression analysis (25), glmnet, ver. 4.0-2, was used to build the model, draw the error distribution and solution paths, and calculate the predictions (26). Optimal Cutpoints, ver. 1.1-4, was used to calculate the AUC and optimal cut-off value (27). For ROC rendering, cutpointr 1.0.32 was used (28). In all analyses, $p<0.05$ was considered statistically significant.

\section{Results}

Histopathological classification of the specimens. Based on the histopathological diagnosis, the thyroid tumors were divided into: i) AdG, ii) FA, iii) PTC, and iv) FTC (Figure 1). There were 41, 20, 68, and four cases of AdG, FA, PTC, and FTC, respectively. Of the 68 PTC cases, $58(85.3 \%)$ were the conventional type and the others included five follicular, four solid, and one cribriform variant. All four cases of FTC were the minimally invasive type without metastasis.

The Vs and VsN measured in benign and malignant tumors. The Vs and VsN in the benign group were $2.12 \pm 0.92 \mathrm{~m} / \mathrm{s}$ and $78.4 \pm 22.7 \%$, respectively, $v s .2 .73 \pm 1.01 \mathrm{~m} / \mathrm{s}$ and $42.3 \pm 26.0 \%$ in the malignant group. Figure 2 shows the representative SWM images of high- and low-elasticity nodules, which are benign and malignant nodules, respectively. Vs was significantly $(p<0.001)$ higher in the malignant group while $\mathrm{VsN}$ was significantly $(p<0.001)$ lower in the malignant group compared to the benign group (Figure 3A and B). AUC represents the diagnostic accuracy. ROC curve analysis of $\mathrm{Vs}$ and $\mathrm{VsN}$ was used to differentiate between benign and malignant nodules, and the AUC was 0.69 for $\mathrm{Vs}$ and 0.85 for $\mathrm{VsN}$ (Figure 3C and D). The cutoff values calculated using Youden's index were $1.97 \mathrm{~m} / \mathrm{s}$ for $\mathrm{Vs}$ and $64.2 \%$ for $\mathrm{VsN}$.

When the cut-off value for Vs was set at $1.97 \mathrm{~m} / \mathrm{s}$, the sensitivity, specificity, positive and, negative predictive values, as well as the diagnostic accuracy were $0.81,0.54$, $0.67,0.70$, and 0.68 , respectively. When the cut-off value for
$\mathrm{VsN}$ was set at $64.2 \%$, the respective measures were 0.81 , $0.77,0.81,0.77$, and 0.79 (Table II).

Analyses of inter-variate relationships and multiple linear regression analysis of $V s N$. The bivariate correlations between the evaluated parameters, which include categorical (sex, age, and the presence of chronic thyroiditis) and continuous (nodule size, Vs, and VsN) variables as explanatory variables, and response (the presence of malignant nodules) as an objective variate, were analyzed and visualized as an inter-variable pairs-combination plot (Figure 4). The peaks of nodule size and $\mathrm{VsN}$ distributions are larger in the benign response group compared to the malignant group. Nodule size was weakly correlated with Vs and $\mathrm{VsN}$ ( $\mathrm{r}=-0.37$ and $\mathrm{r}=0.37$, respectively).

As shown in Figure $3 \mathrm{C}$ and D, ROC curve analysis revealed that $\mathrm{VsN}$ is a better index to discriminate between benign and malignant thyroid tumors than Vs. Therefore, to clarify which factors affect $\mathrm{VsN}$, multiple linear regression analysis was performed. Three continuous variables: i) age, ii) nodule size, and iii) Vs, as well as two categorical variables: i) sex and ii) the presence of chronic thyroiditis and malignant nodule, were selected. Of these, the presence or absence of malignant nodule significantly affected $\mathrm{VsN}$ (Table III). PTC, FTC, AdG, and FA were then compared. The VsN of PTC was significantly lower than that of AdG $(p<0.01)$ and FA $(p<0.01)$. The $\mathrm{VsN}$ of FTC was significantly lower than that of FA $(p<0.02)$ (Figure 5).

Multiple linear regression analysis of VsN in the PTC group. The PTC group accounts for the largest population of thyroid tumors and consists of cases with different individual features. For this reason, we performed correlation analyses with VsN in the PTC group to select variants among the three continuous parameters (age, nodule size, and Vs) and five categorical variables (sex, chronic thyroiditis, extrathyroidal 
A

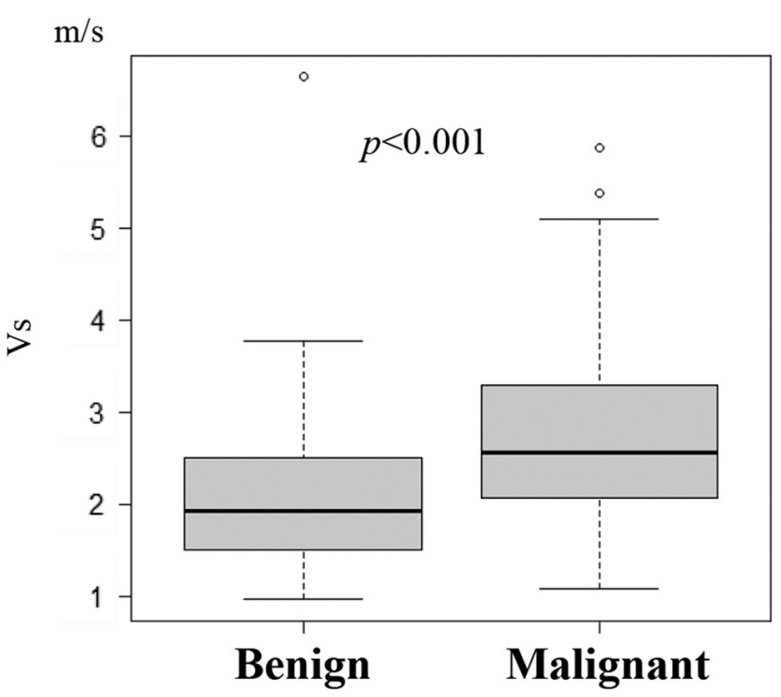

C

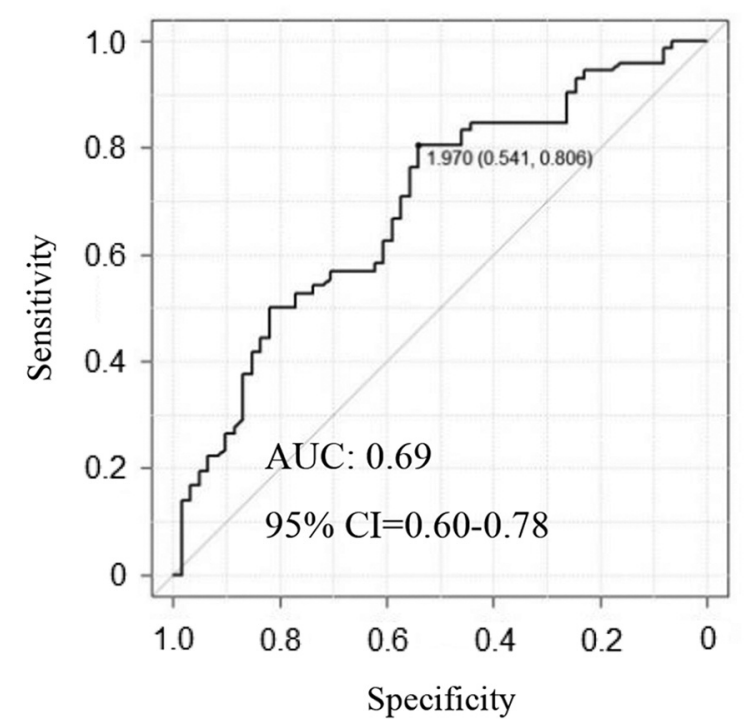

B

VsN

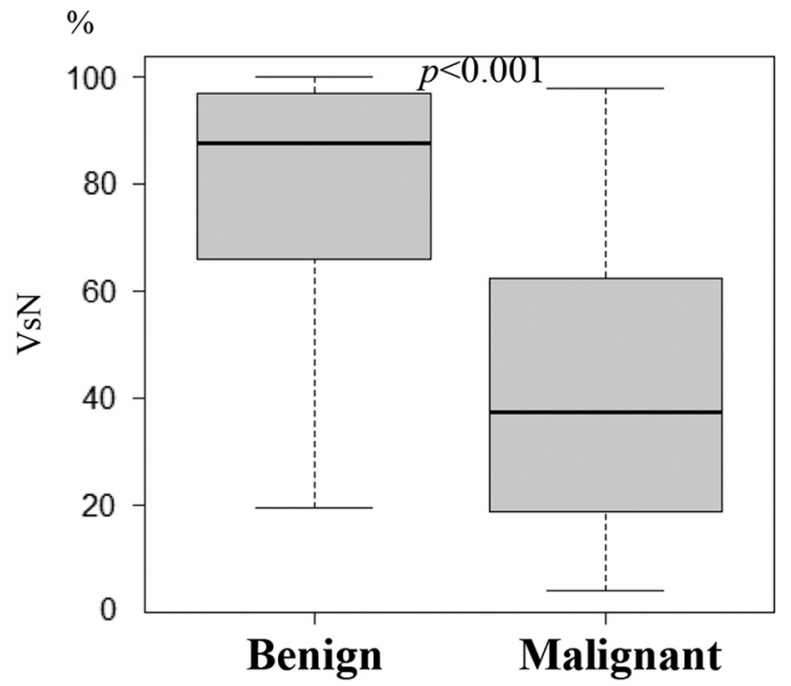

D

VsN

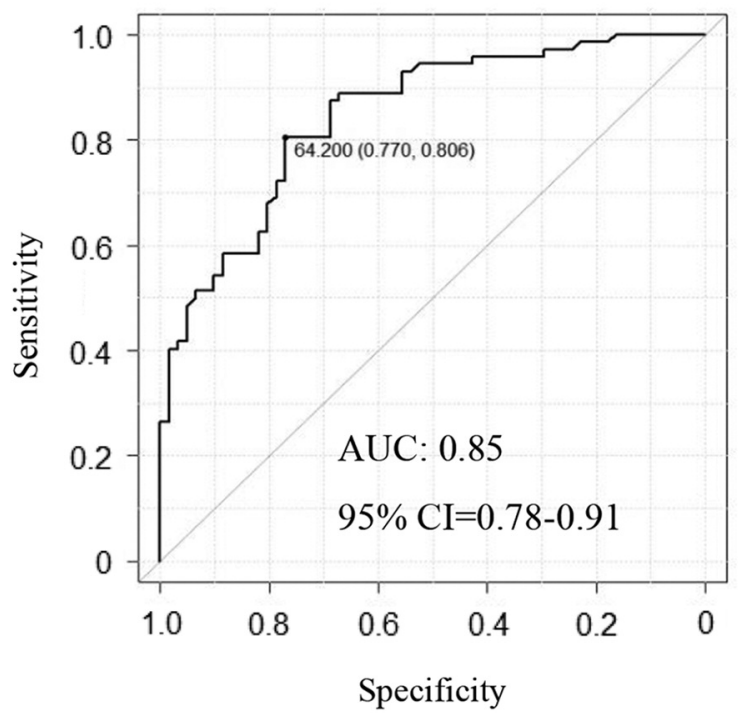

Figure 3. Box-and-whisker plots and ROC curve of Vs and VsN measured in benign and malignant nodules. Shear wave velocity (Vs, A) was significantly higher while the net amount of effective shear wave velocity $(V s N, B)$ was significantly lower in the malignant compared to the benign group. ROC curve analyses of $V s(C)$ and $V s N(D)$ were used to differentiate between benign and malignant nodules.

invasion, multifocal tumor, and lymph node metastasis). Of the eight parameters, nodule size was negatively correlated with $\mathrm{VsN}(\mathrm{r}=-0.48, p<0.0001)$ and PTC with pathological lymph node metastasis had a significantly lower VsN $(p=0.0059)$ (Table IV). In multiple linear regression analysis of $\mathrm{VsN}$, the thyroid nodule size $(p<0.002)$ and lymph node metastasis $(p<0.02)$ were significant predictors of PTC. The adjusted $\mathrm{R}^{2}$ was 0.23 and $p<0.001$ (Table $\mathrm{V}$ ).
Table II. Diagnosis of thyroid nodules with respect to cut-off values in shear wave velocity $(V s)$ and net amount of effective shear wave velocity (VsN).

\begin{tabular}{lcc}
\hline & Malignant & Benign \\
\hline $\mathrm{Vs} \geq 1.97 \mathrm{~m} / \mathrm{s}$ & 58 & 28 \\
$\mathrm{Vs}<1.97 \mathrm{~m} / \mathrm{s}$ & 14 & 33 \\
$\mathrm{VsN} \leq 64.2 \%$ & 58 & 14 \\
$\mathrm{VsN}>64.2 \%$ & 14 & 47 \\
\hline
\end{tabular}




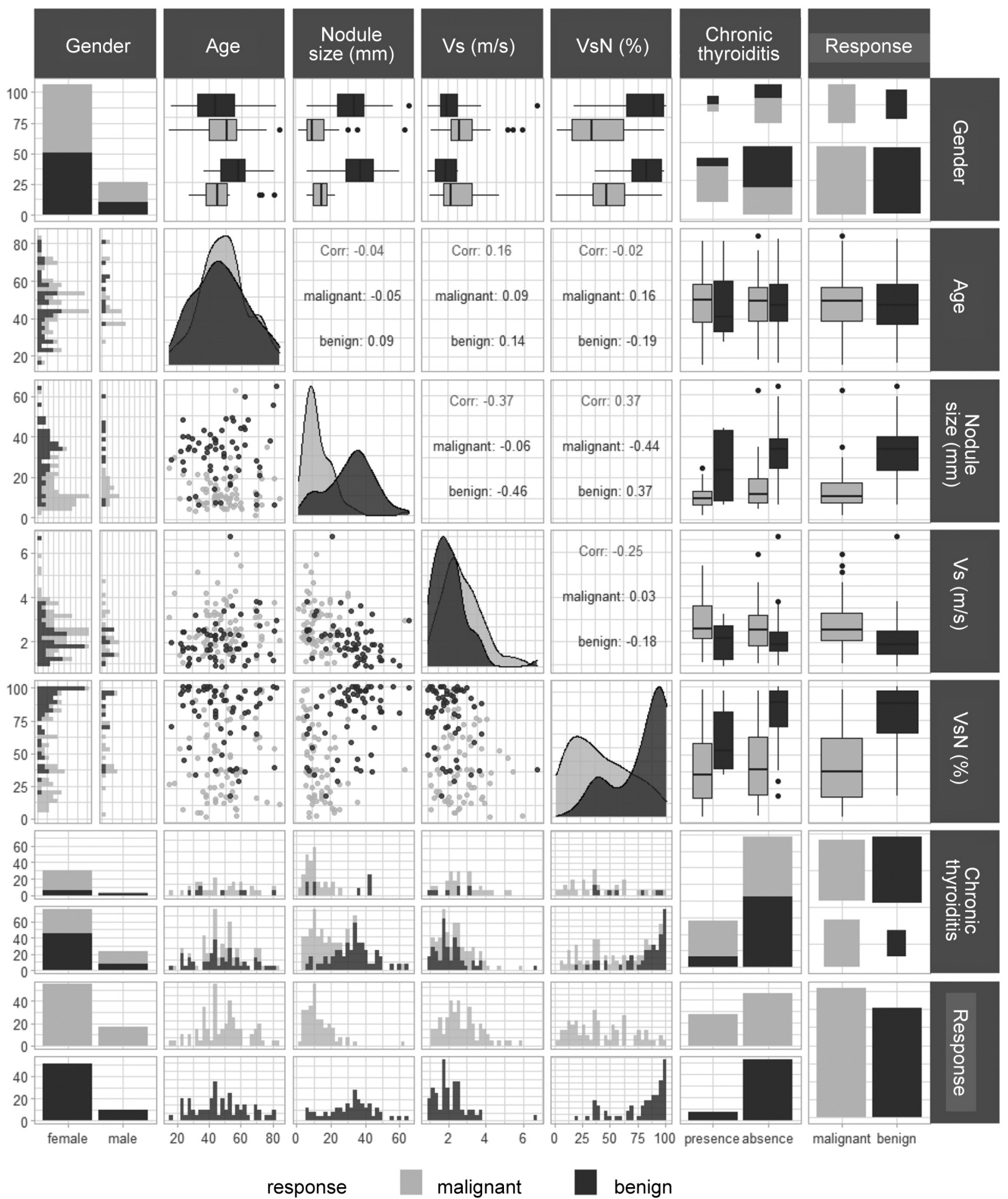

Figure 4. Variables affecting thyroid tumor malignancy. Inter-variable pairs combination plot is shown. On the diagonal, density curves and bar graphs are shown for linear and binary variables, respectively. Scatter charts representing linear relationships, histograms representing the relationship between linear and binary variables, or bar graphs representing the relationship between binary variables are shown below the diagonal. Spearman's correlation coefficients for linear relationships, boxplots representing relationship between linear and binary variables, or mosaic plots representing the relationship between binary variables are shown above the diagonal. Mosaic plots are used to visualize contingency between qualitative variables. The area of each rectangle represents the number of observations. The samples of malignant and benign thyroid nodules are indicated in light gray and black, respectively. Vs: Shear wave velocity; VsN: net amount of effective shear wave velocity. 
in vivo $36: 264-273$ (2022)

Table III. Multivariate linear analysis of the nett amount of effective shear wave velocity (VsN).

\begin{tabular}{|c|c|c|c|c|}
\hline Independent variables & Estimated regression coefficient & Standard error & $\mathrm{t}$ Value & $p$-Value \\
\hline Intercept & 81.31 & 11.92 & 6.82 & $<0.0001$ \\
\hline Age (years) & 0.10 & 0.15 & 0.71 & 0.48 \\
\hline Nodule size (mm) & 0.03 & 0.20 & 0.13 & 0.90 \\
\hline Vs $(\mathrm{m} / \mathrm{s})$ & -2.63 & 2.29 & -1.15 & 0.25 \\
\hline Gender (1: Female, 0: Male) & -2.44 & 5.48 & -0.45 & 0.66 \\
\hline $\begin{array}{l}\text { Chronic thyroiditis } \\
\text { (1: Presence, } 0 \text { : Absence) }\end{array}$ & -8.12 & 5.22 & -1.56 & 0.12 \\
\hline $\begin{array}{l}\text { Malignant nodule } \\
\text { (1: Presence, } 0 \text { : Absence) }\end{array}$ & -32.36 & 5.70 & -5.68 & $<0.0001$ \\
\hline
\end{tabular}

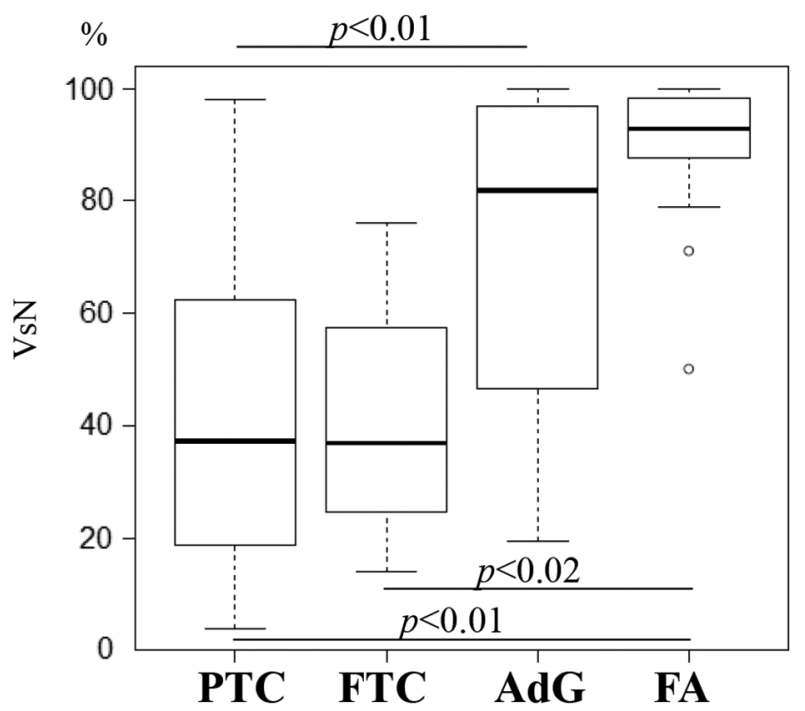

Figure 5. Box-and-whisker plot of the net amount of VsN measured in papillary thyroid carcinoma (PTC), follicular thyroid carcinoma (FTC), adenomatous goitre (AdG), and follicular adenoma (FA). VsN: net amount of effective shear wave velocity.

Data processing to remove the bias in nodule size. In this study, surgery was indicated in the malignant group when malignancy was obvious or suspected following cytology analysis; the size of the malignant nodules had a wide range. Surgery was indicated for benign nodules when the following criteria were met: i) nodule size $>4 \mathrm{~cm}$, ii) growth of the nodule, iii) increased throat discomfort, and iv) the patient requested surgery. The benign nodules tended to be larger than the malignant nodules, and this difference might have influenced the other variables, including VsN. To remove the possible influence of different distributions of nodule size between the two groups, the 133 cases were subjected to stratified sampling based on nodule size. The threshold nodule size was set at $30 \mathrm{~mm}$, which was the size of the largest malignant nodule after excluding outliers, and samples with nodules $\leq 30 \mathrm{~mm}$ were extracted. The correlation coefficient between nodule size and $\mathrm{VsN}$ was -0.07 (Figure 6), which was
Table IV. Characteristics and data analysis for the net amount of effective shear wave velocity $\left(V_{s} N\right)$ in the papillary thyroid carcinoma group.

\begin{tabular}{lccc}
\hline $\begin{array}{l}\text { Independent } \\
\text { variables }\end{array}$ & Mean \pm SD & $\begin{array}{c}\text { Spearman's } \\
\text { rank correlation } \\
\text { coefficient } \\
(\mathrm{r}, p \text {-Value })\end{array}$ & $\begin{array}{c}U \text {-test } \\
(p \text {-Value })\end{array}$ \\
& & $0.16,0.20$ & \\
\hline Age (years) & $49.26 \pm 14.30$ & $-0.48,<0.0001$ & \\
Nodule size (mm) & $12.31 \pm 6.64$ & $0.028,0.82$ & \\
Vs (m/s) & $2.72 \pm 0.99$ & & \\
VsN (\%) & & & \\
Gender: & & & \\
Female (n=52) & $39.86 \pm 26.74$ & & \\
Male (n=16) & $50.43 \pm 22.72$ & & \\
Chronic thyroiditis: & & & \\
$\quad$ Presence (n=27) & $39.41 \pm 26.34$ & & \\
Absence (n=41) & $44.28 \pm 25.98$ & & \\
Extrathyroidal & & & \\
invasion: & & & 0.12 \\
Ex1/2 (n=37) & $37.22 \pm 22.15$ & & \\
Ex0/x (n=31) & $48.46 \pm 29.24$ & & \\
Multifocal tumor: & & & \\
Presence (n=18) & $47.75 \pm 29.40$ & & \\
Absence (n=50) & $40.40 \pm 24.71$ & & \\
Lymph node & & & \\
metastasis: & & & \\
N1a/1b (n=29) & $31.51 \pm 19.43$ & & \\
No/x (n=39) & $50.41 \pm 27.69$ & & \\
\hline
\end{tabular}

SD: Standard deviation.

markedly lower than the value before stratified sampling (0.37) (Figure 4).

Then, a model to discriminate between benign and malignant nodules was constructed using the LASSO method combined with evaluation by leave-one-out cross-validation. This analysis excluded nodule size from the explanatory variables, and only VsN was selected as an explanatory variable to discriminate between benign and malignant nodules. The model calculated AUC as 0.78 (Figure 7A). The VsN values of PTC, FTC, AdG, and FA after stratified sampling (Figure 7B) were similar to the original values (Figure 5). 
Table V. Multivariate linear analysis of the net amount of effective shear wave velocity (VsN) in the papillary thyroid carcinoma group.

\begin{tabular}{|c|c|c|c|c|}
\hline Independent variables & Estimated regression coefficient & Standard error & t Value & $p$-Value \\
\hline Intercept & 66.20 & 60.55 & 10.93 & $<0.0001$ \\
\hline Nodule size (mm) & -1.44 & 0.44 & -3.30 & 0.0016 \\
\hline Lymph node metastasis $(\mathrm{N} 1 \mathrm{a} / \mathrm{b}, \mathrm{No} / \mathrm{x})$ & -14.36 & 5.85 & -2.45 & 0.017 \\
\hline
\end{tabular}

\section{Discussion}

SWM offers qualitative and quantitative advantages over other elastography modalities. Several studies have shown its usefulness for the diagnosis of thyroid nodules. For example, Wu et al. have reported an optimized algorithm of shear-wave elastography showing better reproducibility and performance for the diagnosis of solid thyroid nodules (29). In this study, we examined VsN as a novel index developed to evaluate Vs reliability quantitatively (14). We found a significant difference in the SWM parameters Vs and VsN between malignant and benign thyroid nodules. This is the first report suggesting that $\mathrm{VsN}$ may help diagnose malignant thyroid nodules.

Fukuhara et al. (7) have compared benign to malignant thyroid nodules and reported that the AUC of Vs was 0.81 . In our research, the $\mathrm{Vs}$ of the malignant group was significantly higher than that of the benign group, but the AUC was slightly lower than that in the previous report. Despite this, the AUC of VsN was higher than that of Vs.

Performing SWM in 41 cases of breast carcinoma, Min et al. (30) have reported that a non-numeric Vs value [X.XX], which indicates a value outside the defined range, was output from the device in $63.4 \%$ of these cases. They speculated that the main reason for this was the presence of solid spots that were harder than expected in the target region. Histopathologically, these masses contained abundant fibrous tissue; so, the output X.XX may indicate malignancy $(7,30)$. Based on this, we speculate that the presence of heterogeneous hard tissue in the thyroid is a sign of malignancy. Vs tends to be higher when the target is harder, while $\mathrm{VsN}$ tends to be lower when the variation in $\mathrm{Vs}$ is larger. This might correspond to our unexpected finding of a difference in $\mathrm{VsN}$ between the benign and malignant thyroid tumors, although the correlation between thyroid tissue heterogeneity and $\mathrm{VsN}$ remains unknown and needs further investigation. Recently, Ozturk et al. reported that the preload force affects the shear wave elastography measurements of the thyroid stiffness (31). Although all ultrasound examinations in this study were performed by a single skilled examiner to avoid inter-examiner variability, it is necessary to examine whether $\mathrm{VsN}$ is affected by the preload force.
Chronic thyroiditis may correlate with the degree of fibrosis in the thyroid, which may explain the observed positive correlation with SWM (12). It is thought that fibrosis accelerates the propagation of $\mathrm{Vs}$ and chronic thyroiditis may also affect $\mathrm{VsN}$; however, the multivariate linear analysis of $\mathrm{VsN}$ indicated that chronic thyroiditis was not independently correlated with $\mathrm{VsN}$. Further examination on the relationship between chronic thyroiditis and $\mathrm{VsN}_{\mathrm{S}}$ would be necessary.

Among the six independent variables: i) age, ii) nodule size, iii) Vs, iv) sex, v) chronic thyroiditis, and vi) presence of a malignant nodule, the last one was the only one significantly affecting VsN. The VsN of PTC was significantly lower than that of AdG and FA. Further, the VsN of FTC was significantly lower than that of FA. Although only a few FTC cases were analyzed, our results suggest that $\mathrm{VsN}$ might be a useful parameter for diagnosing FTC, which is currently difficult to differentiate from FA using the available modalities.

We also examined whether SWM is affected by pathological factors. This revealed that nodule size and pathological lymph node metastasis were negatively correlated with VsN in the PTC group. Therefore, it may be possible to predict the presence of lymph node metastasis.

In this study, nodule size was larger in the benign compared to the malignant group. Therefore, we performed multivariate analyses using the LASSO method after stratified sampling to eliminate the bias in nodule size and demonstrated that $\mathrm{VsN}$ can discriminate between benign and malignant nodules.

This study also had some limitations. The tumors analyzed were mostly PTC, while FTC and FA cases were only a few. Therefore, analyses of more FTC and FA nodules are necessary to verify whether SWM can distinguish between FA and FTC.

In conclusion, $\mathrm{VsN}$ is generally considered to be an index used to evaluate the reliability of Vs. However, we found that a low VsN might be a characteristic feature of malignant nodules in the thyroid. SWM parameters, especially VsN, could potentially differentiate between benign and malignant thyroid nodules noninvasively.

\section{Conflicts of Interest}

The Authors have no conflicts of interest to declare. 


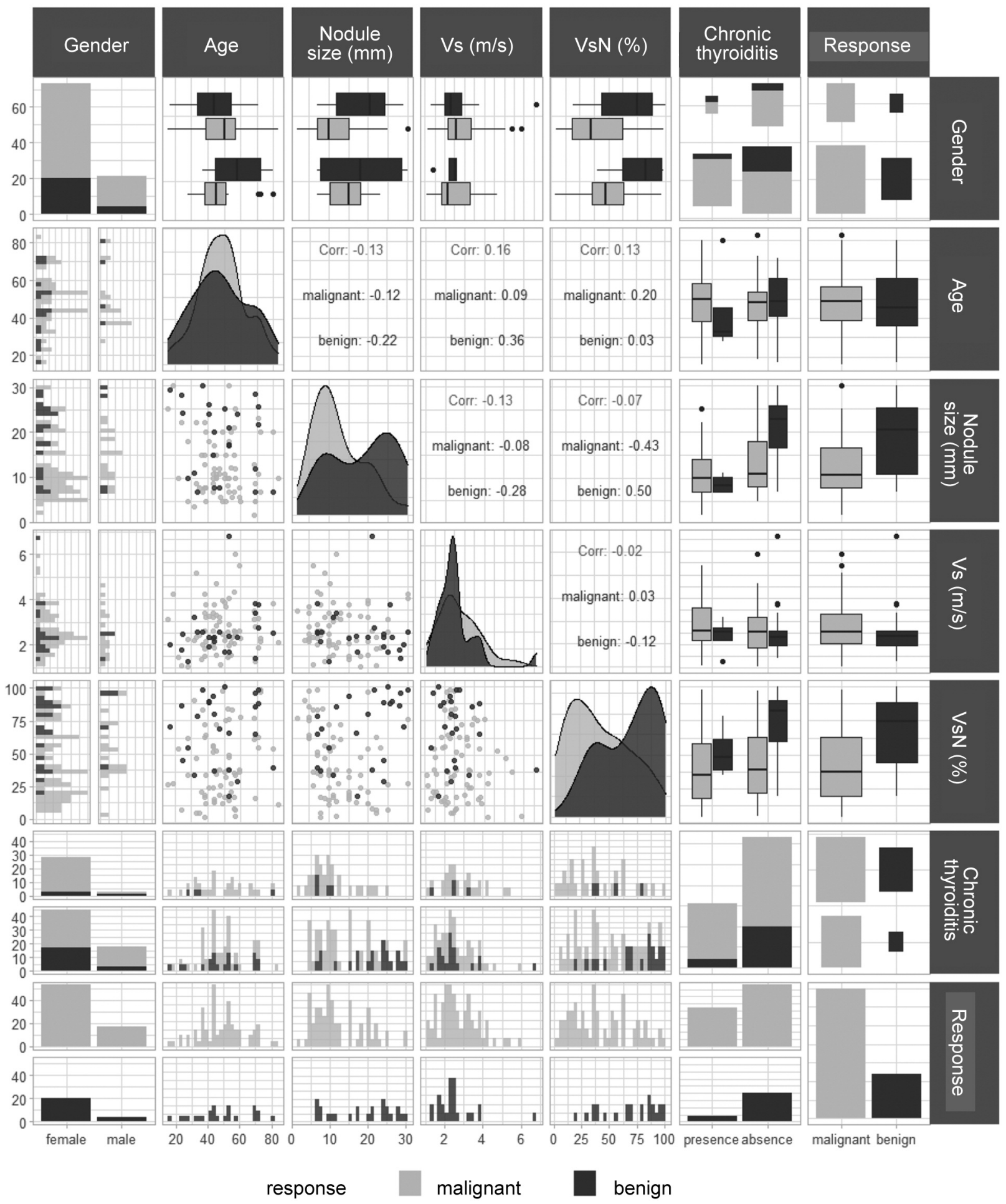

Figure 6. Inter-variable pairs combination plot after stratified sampling. On the diagonal, density curves for linear or bar graphs for binary variables are shown. Scatter charts representing linear relationships, histograms representing relationship between linear and binary variables, or bar graphs representing relationship between binary variables are shown below the diagonal. Spearman's correlation coefficients representing linear relationships, boxplots representing the relationship between linear and binary variables, or mosaic plots representing the relationship between binary variables are shown above the diagonal. Mosaic plots are used to visualize contingency between qualitative variables. The area of each rectangle represents the number of observations. The samples of malignant and benign thyroid nodules are indicated in light gray and black, respectively. Vs: Shear wave velocity; VsN: net amount of effective shear wave velocity. 


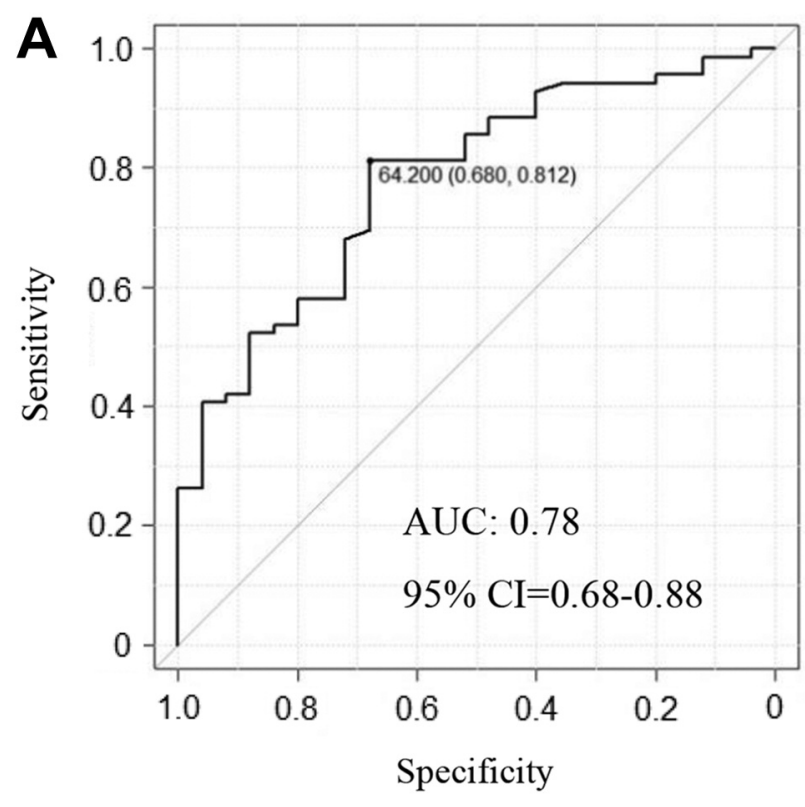

B \%

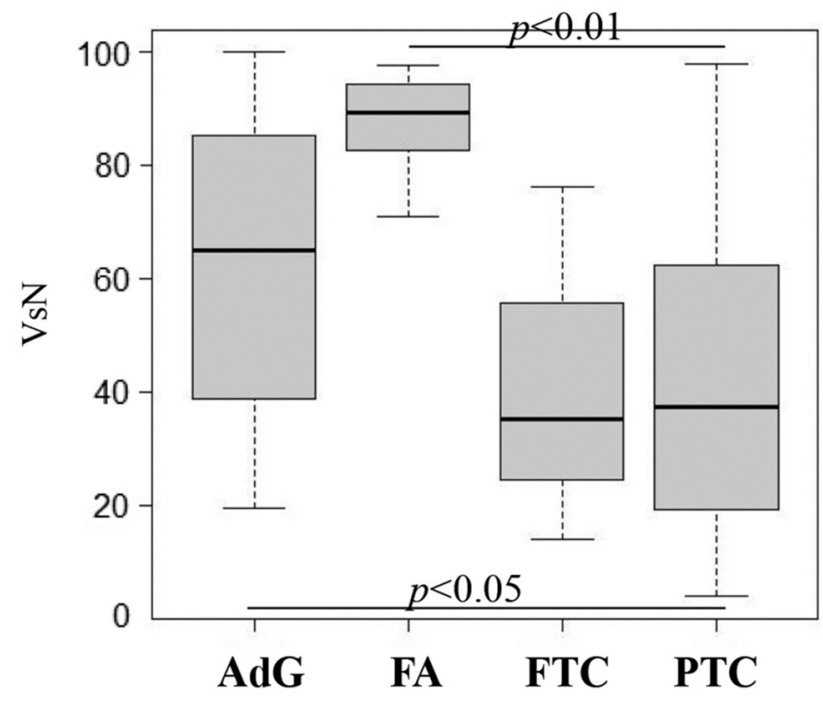

Figure 7. ROC and box-and-whisker plots after stratified sampling. (A) $R O C$ analysis of VSN measured in benign and malignant nodules. (B) A box-and-whisker plot of VsN measured in papillary thyroid carcinoma (PTC), follicular thyroid carcinoma (FTC), adenomatous goitre (AdG), and follicular adenoma (FA). VsN: Net amount of effective shear wave velocity.

\section{Authors' Contributions}

TJ and OI conceptualized and directed this study. TJ and TY obtained ethics approval for this study and performed data collection. TJ and CI performed statistical analyses. TJ and OI wrote the manuscript. SK and IS provided critical suggestions on the manuscript.

\section{Acknowledgements}

We would like to thank Hiroko Tozuka, Yusaku Sato, and Hidemitsu Saito (FUJIFILM Healthcare Co., Ltd., Tokyo, Japan) for their technical advice.

\section{References}

1 Shiina T, Nightingale KR, Palmeri ML, Hall TJ, Bamber JC, Barr RG, Castera L, Choi BI, Chou YH, Cosgrove D, Dietrich CF, Ding H, Amy D, Farrokh A, Ferraioli G, Filice C, FriedrichRust M, Nakashima K, Schafer F, Sporea I, Suzuki S, Wilson S and Kudo M: WFUMB guidelines and recommendations for clinical use of ultrasound elastography: Part 1: basic principles and terminology. Ultrasound Med Biol 41(5): 1126-1147, 2015. PMID: 25805059. DOI: 10.1016/j.ultrasmedbio.2015.03.009

2 Yeh WC, Li PC, Jeng YM, Hsu HC, Kuo PL, Li ML, Yang PM and Lee PH: Elastic modulus measurements of human liver and correlation with pathology. Ultrasound Med Biol 28(4): 467-474, 2002. PMID: 12049960. DOI: 10.1016/s0301-5629(02)00489-1

3 Huwart L, Peeters F, Sinkus R, Annet L, Salameh N, ter Beek LC, Horsmans Y and Van Beers BE: Liver fibrosis: non-invasive assessment with MR elastography. NMR Biomed 19(2): 173179, 2006. PMID: 16521091. DOI: 10.1002/nbm.1030

4 Liao LJ, Chen HW, Hsu WL and Chen YS: Comparison of strain elastography, shear wave elastography, and conventional ultrasound in diagnosing thyroid nodules. J Med Ultrasound 27(1): 26-32, 2019. PMID: 31031532. DOI: 10.4103/JMU.JMU_46_18

5 Zhao CK, Chen SG, Alizad A, He YP, Wang Q, Wang D, Yue WW, Zhang K, Qu S, Wei Q and Xu HX: Three-dimensional shear wave elastography for differentiating benign from malignant thyroid nodules. J Ultrasound Med 37(7): 1777-1788, 2018. PMID: 29315789. DOI: 10.1002/jum.14531

6 Azizi G, Keller JM, Mayo ML, Piper K, Puett D, Earp KM and Malchoff CD: Thyroid nodules and shear wave elastography: a new tool in thyroid cancer detection. Ultrasound Med Biol 41(11): 2855-2865, 2015. PMID: 26277203. DOI: 10.1016/ j.ultrasmedbio.2015.06.021

7 Fukuhara T, Matsuda E, Fujiwara K, Tanimura C, Izawa S, Kataoka $\mathrm{H}$ and Kitano $\mathrm{H}$ : Phantom experiment and clinical utility of quantitative shear wave elastography for differentiating thyroid nodules. Endocr J 61(6): 615-621, 2014. PMID: 24717719. DOI: $10.1507 /$ endocrj.ej14-0061

8 Chang W, Tang L, Lu C, Wu M and Chen M: Shear wave elastography in the evaluation of level VI lymph nodes in papillary thyroid carcinoma: combined with gray-scale ultrasound ex vivo. BMC Cancer 18(1): 1001, 2018. PMID: 30342491. DOI: $10.1186 / \mathrm{s} 12885-018-4897-1$

9 Hefeda MM: Value of the new elastography technique using acoustic radiation force impulse in differentiation between Hashimoto's thyroiditis and Graves' disease. J Clin Imaging Sci 9: 17, 2019. PMID: 31448168. DOI: 10.25259/JCIS-22-2019

10 Bakırtaş Palabıyık F, İnci E, Papatya Çakır ED and Hocaoğlu E: Evaluation of normal thyroid tissue and autoimmune thyroiditis in children using shear wave elastography. J Clin Res Pediatr Endocrinol 11(2): 132-139, 2019. PMID: 30362325. DOI: 10.4274/jcrpe.galenos.2018.2018.0137

11 Kandemirli SG, Bayramoglu Z, Caliskan E, Sari ZNA and Adaletli I: Quantitative assessment of thyroid gland elasticity with shear-wave elastography in pediatric patients with 
Hashimoto's thyroiditis. J Med Ultrason (2001) 45(3): 417-423, 2018. PMID: 29349581. DOI: 10.1007/s10396-018-0859-0

12 Fukuhara T, Matsuda E, Izawa S, Fujiwara K and Kitano H: Utility of shear wave elastography for diagnosing chronic autoimmune thyroiditis. J Thyroid Res 2015: 164548, 2015. PMID: 26257979. DOI: 10.1155/2015/164548

13 Sonoyama T, Murayama N and Inoue N: Development of shear wave measurement with a reliability indicator. Medical Systems Engineering Division 2, Hitachi Aloka Medical, Ltd. 63: 40-44, 2015. Available at: https://hce.fujifilm.com/fileadmin/hitachimedical-eu/product-and-servies/ultrasound/technologies/ Shear_Wave_Measurement_SWM_/MEDIX_Technical_Repor t_-__Development_of_Shear_Wave_Measurement_with_ a_Reliability_Indicator.pdf [Last accessed on October 7, 2021]

14 Yada N, Sakurai T, Minami T, Arizumi T, Takita M, Hagiwara $\mathrm{S}$, Ueshima $\mathrm{K}$, Ida $\mathrm{H}$, Nishida $\mathrm{N}$ and Kudo $\mathrm{M}$ : A newly developed shear wave elastography modality: with a unique reliability index. Oncology 89 Suppl 2: 53-59, 2015. PMID: 26580548. DOI: 10.1159/000440632

15 Bychkov A: World Health Organization (WHO) classification. PathologyOutlines.com. Available at: https://www.pathology outlines.com/topic/thyroidwho.html [Last accessed on October $7,2021]$

16 Lloyd RV, Osamura RY, Klöppel G and Rosai J: WHO classification of tumours of the thyroid gland. In: WHO Classification of Tumours of Endocrine Organs. WHO/IARC Classification of Tumours $4^{\text {th }}$ Edition, Lyon, IARC Publications, pp. 66, 2017.

17 Baloch ZW, Cibas ES, Clark DP, Layfield LJ, Ljung BM, Pitman MB and Abati A: The National Cancer Institute Thyroid fine needle aspiration state of the science conference: a summation. Cytojournal 5: 6, 2008. PMID: 18394201. DOI: 10.1186/17426413-5-6

18 Cibas ES, Ali SZ and NCI Thyroid FNA State of the Science Conference: The Bethesda system for reporting thyroid cytopathology. Am J Clin Pathol 132(5): 658-665, 2009. PMID: 19846805. DOI: 10.1309/AJCPPHLWMI3JV4LA

19 Ali SZ and Cibas ES: The Bethesda system for reporting thyroid cytopathology: definitions, criteria and explanatory notes. Springer, New York, 2018. DOI: 10.1007/978-3-319-60570-8

20 Cancer.Net: Thyroid Cancer Statistics. Available at: https:// www.cancer.net/cancer-types/thyroid-cancer/statistics [Last accessed on October 7, 2021]

21 Kanda Y: Investigation of the freely available easy-to-use software 'EZR' for medical statistics. Bone Marrow Transplant 48(3): 452-458, 2013. PMID: 23208313. DOI: 10.1038/bmt. 2012.244
22 R Core Team (2018) R: A language and environment for statistical computing. R Foundation for Statistical Computing, Vienna, Austria. Available at: https://www.r-project.org [Last accessed on October 7, 2021]

23 psych: Procedures for Psychological, Psychometric, and Personality Research. Available at: https://cran.r-project.org/web/ packages/psych/index.html [Last accessed on October 7, 2021]

24 plsdepot: Partial least squares (PLS) data analysis methods. Available at: https://cran.r-project.org/web/packages/plsdepot/ index.html [Last accessed on October 7, 2021]

25 Hastie T, Tibshirani R and Wainwright M: The Lasso for linear models. In: Statistical Learning with Sparsity: The Lasso and Generalizations. Boca Raton, CRC Press, 2015.

26 glmnet: Lasso and elastic-net regularized generalized linear models. Available at: https://cran.r-project.org/web/packages/ glmnet [Last accessed on October 7, 2021]

27 OptimalCutpoints: Computing optimal cutpoints in diagnostic tests. Available at: https://cran.r-project.org/web/packages/ OptimalCutpoints/index.html [Last accessed on October 7, 2021]

28 cutpointr: Determine and evaluate optimal cutpoints in binary classification tasks. Available at: https://cran.r-project.org/ web/packages/cutpointr/index.html [Last accessed on October 7, 2021]

$29 \mathrm{Wu} \mathrm{H}$, Chen Q, Liu Y, Chen J and Deng W: Optimized algorithm in solid thyroid nodule elastography. Oncol Lett 20(5): 248, 2020. PMID: 32994811. DOI: 10.3892/ol.2020.12111

30 Bai M, Du L, Gu J, Li F and Jia X: Virtual touch tissue quantification using acoustic radiation force impulse technology: initial clinical experience with solid breast masses. J Ultrasound Med 31(2): 289-294, 2012. PMID: 22298873. DOI: 10.7863/ jum.2012.31.2.289

31 Ozturk A, Zubajlo RE, Dhyani M, Grajo JR, Mercaldo N, Anthony BW and Samir AE: Variation of shear wave elastography with preload in the thyroid: quantitative validation. J Ultrasound Med 40(4): 779-786, 2021. PMID: 32951229. DOI: 10.1002/jum. 15456
Received August 16, 2021

Revised October 8, 2021

Accepted October 13, 2021 